\title{
Black-hole thermodynamics with modified dispersion relations and generalized uncertainty principles
}

\author{
Giovanni AMELINO-CAMELIA ${ }^{a}$, Michele ARZANO $^{b}$, \\ Yi LING ${ }^{c}$ and Gianluca MANDANICI ${ }^{a}$ \\ ${ }^{a}$ Dip. Fisica Univ. Roma "La Sapienza" and Sez. Roma1 INFN, \\ Piazzale Moro 2, Roma, Italy \\ ${ }^{b}$ Institute of Field Physics, Dept Physics and Astronomy \\ University of North Carolina, Chapel Hill, NC 27599, USA \\ ${ }^{c}$ Center for Gravity and Relativity, Department of Physics, \\ Nanchang University, Nanchang 330047, China
}

\begin{abstract}
SUMMARY
In several approaches to the quantum-gravity problem evidence has emerged of the validity of a "GUP" (a Generalized position-momentum Uncertainty Principle) and/or a "MDR" (a modification of the energy-momentum dispersion relation), but very little is known about the implications of GUPs and MDRs for black-hole thermodynamics, another key topic for quantum-gravity research. We investigate an apparent link, already suggested in an earlier exploratory study involving two of us, between the possibility of a GUP and/or a MDR and the possibility of a log term in the area-entropy black-hole formula. We then obtain, from that same perspective, a modified relation between the mass of a black hole and its temperature, and we examine the validity of the "Generalized Second Law of black-hole thermodynamics" in theories with a GUP and/or a MDR. After an analysis of GUP-and MDR-modifications of the black-body radiation spectrum, we conclude the study with a description of the black-hole evaporation process.
\end{abstract}

\section{Introduction}

Various arguments suggest that the description of black holes should be an important aspect of a quantum-gravity theory, and that some key operatively-meaningful (not merely formal) differences between alternative theories should emerge as we establish, within each approach, how the singularity, the evaporation, the "thermodynamics", and the "information paradox" are handled. A similar role could be played, as stressed in the recent literature [1, 2, 3, 4, by the analysis of the energy-momentum dispersion relation and the position-momentum uncertainty principle. Different approaches to the quantum-gravity problem lead to different expectations for what concerns the possibility of a MDR (a modified energy-momentum dispersion relation) and the possibility of a GUP (a generalized positionmomentum uncertainty principle). In particular, in the study of Loop Quantum Gravity and of models based on noncommutative geometry there has been strong interest [5, 6, 7] in some candidate modifications of the energy-momentum dispersion relation. Generalized uncertainty principles [8, 9] have been considered primarily in the literature on String Theory [10] and on models based on noncommutative geometry [11. The form of the energy-momentum dispersion relation and of the position-momentum uncertainty relation can therefore be used to characterize alternative approaches to the quantumgravity problem. 
Two of us were recently involved [12] in research exploring a possible link between the predictions that a quantum-gravity theory makes for black-hole thermodynamics and the predictions that the same theory makes for the energy-momentum dispersion relation and the position-momentum uncertainty relation. By establishing the nature of such a link one would, in our opinion, obtain a valuable characterization of the type of internal logical consistency that various aspects of a quantum-gravity theory should satisfy. The study reported in Ref. [12] was not the first to explore the possible role of MDRs and/or GUPs for black holes, as we discuss in greater detail here in Section 9 where we comment of some relevant references [13, 14, 15, 16, 17, 18, but all of these studies, including Ref. [12, focused on one or another aspect of the possible interplay between MDR/GUP results and black holes, without attempting to obtain a wider picture. We here work in the spirit of Ref. [12, but we attempt to give the first elements of a general analysis of some key characteristics of black-hole physics, as affected by some scenarios for a MDR or a GUP.

Sections 2, 3 and 4, set the stage, by reviewing some results in the MDR/GUP literature and revisiting the point already made in Ref. 12, which concerns an apparent link between the log-area terms in the entropy-area relation for black holes and certain formulations of the MDR and the GUP. In Section 5 we explore, still within the working assumptions adopted in Ref. [12, the implications of a MDR and/or a GUP for the Bekenstein entropy bound and for the Generalized Second Law of thermodynamics. We find that the implications are significant and we conjecture that they should also not be negligible in the analysis of other entropy-bound proposals. Section 6 considers a role for $\mathrm{MDR} / \mathrm{GUP}$ modifications in the analysis of the black-body radiation spectrum, and again exposes some significant changes with respect to the standard picture, including the possibility that the characteristic frequency of black-body radiation at given temperature $T$ might have a dependence on $T$ such that in the infinite-temperature limit the characteristic frequency would take a finite (Planckian) value. It is then perhaps not surprising that in the analysis of the black-hole evaporation process, discussed in Section 7, we also find some characteristic MDR/GUP-induced new features, such as the possibility that the energy flux emitted by the black hole might diverge when the black-hole mass reaches a certain finite (Planckian) value. In Section 8, we comment on one key aspect which might deserve further consideration: for these theories with MDRs and/or GUPs there has been some speculation that the speed of massless particles might be different from the familiar speed-of-light scale value of $c$. In Sections 1-7 we assume throughout that $c$ still is the speed of massless particles, but in Section 8 we establish how the analysis of black-body radiation would be changed if one implemented some alternatives considered in the literature. In Section 9 we compare our analysis with other studies which have considered the implications of a MDR or a GUP for some aspects of black-hole physics. Section 10 concludes the paper with some remarks on the outlook of this research programme.

\section{MDRs and GUPs in Quantum Gravity and implications for a Planck-scale particle-localization limit}

\subsection{MDRs and GUPs in Quantum Gravity}

In the study of the Quantum-Gravity problem the emergence of modified energy-momentum relations and/or generalized position-momentum uncertainty principles, although of course not guaranteed, can be motivated on general grounds, and also finds support in the direct analysis of certain QuantumGravity scenarios.

The hypothesis of modified energy-momentum dispersion relations is understandably popular among those adopting a "spacetime foam" intuition in the study of the quantum-gravity problem, especially when an analogy between spacetime foam and some more familiar forms of medium (such as certain crystal structures of interest in condensed-matter studies) is proposed. It is then expected that wave dispersion "in vacuo" (in the spacetime foam) might resemble wave dispersion in other media. A modified dispersion relation can also be favoured by the expectation, shared by many researchers of the field, that the Planck length might fundamentally set the minimum allowed value for wavelengths. A nonlinear relationship between energy and (space-) momentum can be easily adjusted 
in such a way that in the infinite-energy limit the momentum saturates to the Planck-scale value (and wavelength saturates to the Planck-length value). This possibility has become more attractive with the recent realization 19, 20, 21 that a modified energy-momentum dispersion relation can also be introduced as an observer-independent $l^{1}{ }^{1}$, in which case the Planckian minimum-wavelength hypothesis can be introduced as a physical law valid in every frame. The analysis of some quantumgravity scenarios, even in cases in which the emergence of modified energy-momentum relations was not intended in the original setup of the framework, has shown some explicit mechanisms for the emergence of modified dispersion relations. This is particularly true of some approaches based on noncommutative geometry [7, 20] and within the Loop-Quantum-Gravity approach [5, 6]. In most cases one is led to consider a dispersion relation of the type ${ }^{2}$

$$
\vec{p}^{2}=f\left(E, m ; L_{p}\right) \simeq E^{2}-\mu^{2}+\alpha_{1} L_{p} E^{3}+\alpha_{2} L_{p}^{2} E^{4}+O\left(L_{p}^{3} E^{5}\right),
$$

where $f$ is the function that gives the exact dispersion relation, and on the right-hand side we just assumed the applicability of a Taylor-series expansion for $E \ll 1 / L_{p}$. The coefficients $\alpha_{i}$ can take different values in different Quantum-Gravity proposals.

The fact that these Planck-scale-deformed dispersion relations may have observably large consequences in some (however rare) physical contexts has led to interest in this research also from the perspective of phenomenology [1, 22, 23, 24, 25].

The situation concerning the possibility of a generalized position-momentum uncertainty principle is rather similar. On general grounds it can be motivated by the intuition [8, 9] that the solution of the quantum-gravity problem might require the introduction of an absolute Planckian limit on the size of the collision region, applicable to high-energy microscopic collision processes. For example, a GUP of the form

$$
\delta x \geq \frac{1}{\delta p}+\alpha L_{p}^{2} \delta p+O\left(L_{p}^{3} \delta p^{2}\right),
$$

which has been derived within the String Theory approach to the quantum-gravity problem [10], is such that at small $\delta p$ one finds the standard dependence of $\delta x$ on $\delta p$ ( $\delta x$ gets smaller as $\delta p$ increases) but for large $\delta p$ the Planckian correction term becomes significant and keeps $\delta x \geq L_{p}$. Within String Theory the coefficient $\alpha$ should take a value of roughly the ratio between the square of the string length and the square of the Planck length, but this of course might work out differently in other Quantum-Gravity proposals.

While in the parametrization of (10) we included a possible correction term suppressed only by one power of the Planck length, in (2) such a linear-in- $L_{p}$ is assumed not to be present. This reflects the status of the presently-available literature: for the MDR a large number of alternative formulations, including some with the linear-in- $L_{p}$ term, are being considered, as they find support in different approaches to the quantum-gravity problem (and different preliminary results adopting alternative approximation schemes within a given approach), whereas all the discussions of a GUP assume that the leading-order correction should be proportional to the square of $L_{p}$.

\subsection{MDR and a Planck-scale particle-localization limit}

The analysis reported in Ref. [12] exposed a previously unnoticed common feature of MDR and GUP scenarios. This has to do with a Planck-scale limit on the localization of a particle, and an associated modification of the Bekenstein argument for a area-entropy black-hole relation.

Arguably the closest starting point for the construction of the correct Quantum Gravity should be Quantum Field Theory, and within Quantum Field Theory the most striking quantum effect concerns

\footnotetext{
${ }^{1}$ But this usually requires introducing a nonlinear deformation of the action of Lorentz boosts.

${ }^{2}$ We denote with $m$, as conventional, the rest energy of the particle. The mass parameter $\mu$ on the right-hand side is directly related to the rest energy, but $\mu \neq m$ if the $\alpha_{i}$ do not all vanish. For example, if $\alpha_{1} \neq 0$ but $\alpha_{i}=0$ for every $i \geq 2$ one of course obtains $\mu^{2}=m^{2}+\alpha_{1} L_{p} m^{3}$. This needed to be clarified since it is relevant for more general analyses of MDRs, but in our study we are always concerned with particles which are either massless or anyway are analyzed at energies such that the mass can be neglected, and therefore both $\mu$ and $m$ will never actually enter our key formulas.
} 
an absolute limit on the localization of a particle of energy $E$, codified in the relation $E \geq \frac{1}{\delta x}$. While in nonrelativistic quantum mechanics a particle of any energy can always be sharply localized (at the price of renouncing to all information on the conjugate momentum), within Quantum Field Theory only in the infinite-energy limit a particle can be sharply localized. And among those studying the quantum-gravity problem one frequently encounters the intuition that at the Quantum-Gravity level the idealization of sharp localization should disappear completely.

In the spirit of Ref. 12] one can attempt to codify this quantum-gravity intuition in a relation of the type

$$
E \geq \frac{1}{\delta x}\left(1-\Delta\left(L_{p}, \delta x\right)\right)
$$

where $\Delta$ is some function of $L_{p}$ and $\delta x$, perhaps such that $E \rightarrow \infty$ already at some finite value of $\delta x$ (so that the idealization $\delta x \rightarrow 0$ is excluded). And it was observed in Ref. 12 that both the idea of a MDR and the idea of a GUP would support a formula of the type (3), with nonzero $\Delta$.

Let us briefly review this analysis reported in Ref. 12, starting with the case of a MDR of the type (11). We can follow the familiar derivation 32] of the relation $E \geq \frac{1}{\delta x}$, substituting, where necessary, the standard special-relativistic dispersion relation with its Planck-scale modified version. It is convenient to start by focusing on the case of a particle of mass $M$ at rest, whose position is being measured by a procedure involving a collision with a photon of energy $E_{\gamma}$ and momentum $p_{\gamma}$. According to Heisenberg's uncertainty principle, in order to measure the particle position with precision $\delta x$ one should use a photon with momentum uncertainty $\delta p_{\gamma} \geq \frac{1}{\delta x}$. Following the standard argument [32, one takes this $\delta p_{\gamma} \geq \frac{1}{\delta x}$ relation and converts it into the relation $\delta E_{\gamma} \geq \frac{1}{\delta x}$ using the special relativistic dispersion relation. Finally $\delta E_{\gamma} \geq \frac{1}{\delta x}$ is converted into the relation $M \geq \frac{1}{\delta x}$ because the measurement procedure requires $\delta E \leq M$, in order to ensure that the relevant energy uncertainties are not large enough to allow the production of additional copies of the particle whose position is being measured.

If indeed our Quantum-Gravity scenario hosts a Planck-scale modification of the dispersion relation of the form (11) then clearly the relation between $\delta p_{\gamma}$ and $\delta E_{\gamma}$ should be re-written as follows

$$
\delta p_{\gamma} \simeq\left(1+\alpha_{1} L_{p} E+3\left(\frac{\alpha_{2}}{2}-\frac{\alpha_{1}^{2}}{8}\right) L_{p}^{2} E^{2}\right) \delta E_{\gamma}
$$

which then leads to the requirement

$$
M \geq \frac{1}{\delta x}-\alpha_{1} \frac{L_{p}}{(\delta x)^{2}}+\left(\frac{11}{8} \alpha_{1}^{2}-\frac{3}{2} \alpha_{2}\right) \frac{L_{p}^{2}}{(\delta x)^{3}}+O\left(\frac{L_{p}^{3}}{(\delta x)^{4}}\right) .
$$

These results strictly apply to the measurement of the position of a particle at rest, but they can be straightforwardly generalized [32 (simply using a boost) to the case of the measurement of the position of a particle of energy $E$. For the standard case this leads to the $E \geq 1 / \delta x$ relation while in presence of an MDR one easily finds

$$
E \geq \frac{1}{\delta x}-\alpha_{1} \frac{L_{p}}{(\delta x)^{2}}+\left(\frac{11}{8} \alpha_{1}^{2}-\frac{3}{2} \alpha_{2}\right) \frac{L_{p}^{2}}{(\delta x)^{3}}+O\left(\frac{L_{p}^{3}}{(\delta x)^{4}}\right) .
$$

\subsection{GUP and a Planck-scale particle-localization limit}

While the connection between a MDR and a Planck-scale particle-localization limit is somewhat less obvious (and in fact we found no mention of it in the literature previous to Ref. 12]), it is not at all surprising that the GUP would give rise to such a particle-localization limit. In fact, as mentioned, the GUP is primarily viewed as a way to introduce a Planckian limit on the size of the collision region, applicable to high-energy microscopic collision processes, and a limitation on the size of collision regions would naturally be expected to lead to a particle-localization limit. Indeed, as the careful reader can easily verify, from the GUP one obtains (following again straightforwardly the familiar 
line of analysis discussed in Ref. [32]) a modification of the relation $E \geq 1 / \delta x$. The modification is of the type $E \geq 1 / \delta x+\Delta$, with $\Delta$ of order $\alpha L_{p}^{2} / \delta x^{3}$, and originates from the fact that according to the GUP, (2), one obtains $\delta p_{\gamma} \geq 1 / \delta x+\lambda_{s}^{2} / \delta x^{3}$ (instead of the original $\delta p_{\gamma} \geq 1 / \delta x$ ). Using the standard special-relativistic dispersion relation for a photon $p_{\gamma}=E_{\gamma}$ the condition on the momentum uncertainty translates in a condition on the energy uncertainty $\delta E_{\gamma} \geq \frac{1}{\delta x}\left(1+\alpha \frac{L_{p}^{2}}{\delta x^{2}}\right)$, and ultimately this leads to

$$
E \geq \frac{1}{\delta x}+\alpha \frac{L_{p}^{2}}{(\delta x)^{3}}+O\left(\frac{L_{p}^{3}}{(\delta x)^{4}}\right)
$$

\section{MDR and black hole entropy}

In this section we revisit the argument already proposed in Ref. [12, suggesting that a Planck-scale modification of the particle-localization limit, of the type (6) or (7), can be used to motivate corrections to the $S=A /\left(4 L_{p}^{2}\right)$ area-entropy relation for black holes. We focus here on the case of a MDR, but since the key ingredient is the Planck-scale particle-localization limit, one should expect that, as we confirm explicitly in the next section, the same line of analysis is applicable also to the case in which one takes as starting point a GUP. Since the literature on MDRs is composed both of papers using arguments that are only sufficient to specify the first terms in a series expansion of the MDR at energies below the Planck scale, and some analyses proposing a complete all-order formula for the MDR, we find appropriate to consider these possibilities separately. The power-series analysis will already show that the implications of a MDR can be significant. But the power-series analysis can only be reliably used at energies safely below the Planck scale. In considering some examples of allorder MDR proposals we will also develop some intuition for the type of implications that a MDR could have for black-hole physics at Planckian energy scales.

Our argument connecting a MDR (a particle-localization limit) and some modifications of the areaentropy relation for black holes is formulated in a scheme of analysis first introduced by Bekenstein [27, which is actually one of the classic arguments for the description of the entropy-area relation. In order to render our presentation self-contained we open this section by describing this classic Bekenstein argument, but we just sketch out the Bekenstein derivation since we expect most readers to be already familiar with it.

\subsection{The original Bekenstein argument (with unmodified dispersion relation and unmodified uncertainty principle)}

The argument presented by Bekenstein in Ref. 27] uses very simple ingredients to suggest that the entropy of a black hole should be proportional to its (horizon-surface) area. The starting point is the general-relativity result [28] establishing that the minimum increase of area when the black hole absorbs a classical particle of energy $E$ and size $s$ is $\Delta A \simeq 8 \pi L_{p}^{2} E s$ (in "natural units" with $\hbar=c=1$ ). In order to describe the absorption of a quantum particle one must describe the size of the particle in terms of the uncertainty in its position [27, 29], $s \sim \delta x$, and take into account a "calibration factor" [30, 31, 12] $(\ln 2) / 2 \pi$ that connects the $\Delta A \geq 8 \pi L_{p}^{2} E s$ classical-particle result with the quantum-particle estimate $\Delta A \geq 4(\ln 2) L_{p}^{2} E \delta x$. Bekenstein then enforces the requirement that a particle with position uncertainty $\delta x$ should at least [32] have energy $E \sim 1 / \delta x$, which leads to $\Delta A \geq 4(\ln 2) L_{p}^{2}$, and assumes that the entropy depends only on the area of the black hole. Also using the fact that the minimum increase of entropy should be $\ln 2$, independently of the value of the area, one then concludes that

$$
\frac{d S}{d A} \simeq \frac{\min (\Delta S)}{\min (\Delta A)} \simeq \frac{\ln 2}{4(\ln 2) L_{p}^{2}} .
$$

From this it follows that (up to an irrelevant constant contribution to entropy):

$$
S \simeq \frac{A}{4 L_{p}^{2}}
$$




\subsection{MDR and black hole entropy in leading order}

The Bekenstein argument implicitly assumes (through the $E \geq 1 / \delta x$ relation) that the energymomentum dispersion relation and the position-momentum uncertainty principle take the standard form. Let us now reformulate the argument, still assuming a standard form for the position-momentum uncertainty principle, but introducing a MDR of the type (11). As in the original Bekenstein argument [27, we take as starting point the general-relativity result which establishes that the area of a black hole changes according to $\Delta A \geq 8 \pi L_{p}^{2} E s$ when a classical particle of energy $E$ and size $s$ is absorbed. And again we describe the size of the particle in terms of the uncertainty in its position as done in the previous subsection, obtaining $\Delta A \geq 4(\ln 2) L_{p}^{2} E \delta x$. Whereas in the original Bekenstein argument one then enforces the relation $E \geq 1 / \delta x$ (and this leads to $\Delta A \geq 4(\ln 2) L_{p}^{2}$ ), we must take into account the MDR-induced Planck-length modification in (6), obtaining

$$
\begin{aligned}
\Delta A & \geq 4(\ln 2)\left[L_{p}^{2}-\frac{\alpha_{1} L_{p}^{3}}{\delta x}-\frac{\left(\frac{3}{2} \alpha_{2}-\frac{11}{8} \alpha_{1}^{2}\right) L_{p}^{4}}{(\delta x)^{2}}\right] \\
& \simeq 4(\ln 2)\left[L_{p}^{2}-\frac{\alpha_{1} L_{p}^{3}}{R_{S}}-\frac{\left(\frac{3}{2} \alpha_{2}-\frac{11}{8} \alpha_{1}^{2}\right) L_{p}^{4}}{\left(R_{S}\right)^{2}}\right] \\
& \simeq 4(\ln 2)\left[L_{p}^{2}-\frac{\alpha_{1} 2 \sqrt{\pi} L_{p}^{3}}{\sqrt{A}}-\frac{\left(\frac{3}{2} \alpha_{2}-\frac{11}{8} \alpha_{1}^{2}\right) 4 \pi L_{p}^{4}}{A}\right],
\end{aligned}
$$

where we also used the fact that in falling in the black hole the particle acquires [15, 33, 34 position uncertainty $\delta x \sim R_{S}$, where $R_{S}$ is the Schwarzschild radius (and of course $A=4 \pi R_{S}^{2}$ ). From (11) we derive an area-entropy relation assuming that the entropy of the black hole depends only on its area and that the minimum increase of entropy should be, independently of the value of the area, $\ln 2$ :

$$
\frac{d S}{d A} \simeq \frac{\min (\Delta S)}{\min (\Delta A)} \simeq \frac{\ln 2}{4(\ln 2) L_{p}^{2}\left[1-\frac{\alpha_{1} 2 \sqrt{\pi} L_{p}}{\sqrt{A}}-\frac{\left(\frac{3}{2} \alpha_{2}-\frac{11}{8} \alpha_{1}^{2}\right) 4 \pi L_{p}^{2}}{A}\right]} \simeq\left(\frac{1}{4 L_{p}^{2}}+\frac{\alpha_{1} \sqrt{\pi}}{2 L_{p} \sqrt{A}}+\frac{\left(\frac{3}{2} \alpha_{2}-\frac{11}{8} \alpha_{1}^{2}\right) \pi}{A}\right)
$$

which gives (up to an irrelevant constant contribution to entropy)

$$
S \simeq \frac{A}{4 L_{p}^{2}}+\alpha_{1} \sqrt{\pi} \frac{\sqrt{A}}{L_{p}}+\left(\frac{3}{2} \alpha_{2}-\frac{11}{8} \alpha_{1}^{2}\right) \pi \ln \frac{A}{L_{p}^{2}} .
$$

This result of course reproduces the famous linear formula if all coefficients $\alpha_{i}$ vanish. If the cubic term $\alpha_{1} E^{3}$ is present in the energy-momentum dispersion relation then the leading correction goes like $\sqrt{A}$, whereas if the first nonzero coefficient in the dispersion relation expansion is $\alpha_{2}$ the leading correction term goes like $\log A$. Our "improved Bekenstein argument" therefore provides a possible link between the form of the MDR (and of the GUP, as we stress later) and the all-order form of the entropy-area relation for black holes. For example, if within a given quantum-gravity approach one can find a general argument suggesting that there are no $\sqrt{A}$ terms in the entropy-area relation, then one can use our improved Bekenstein argument to deduce that within that given quantum-gravity approach one should not find terms of the type $\alpha_{1} E^{3}$ in the energy-momentum dispersion relation.

Over the last few years both in String Theory and in Loop Quantum Gravity some techniques for the direct analysis of the entropy of black holes, using their quantum properties, have been developed, and these techniques are now able [35, 36] to go even beyond the entropy-area-proportionality contribution: they establish that the leading correction should be of log-area type, so that one expects (for $A \gg L_{p}^{2}$ ) an entropy-area relation for black holes of the type

$$
S=\frac{A}{4 L_{p}^{2}}+\rho \ln \frac{A}{L_{p}^{2}}+O\left(\frac{L_{p}^{2}}{A}\right)
$$


where $\rho$ is a coefficient which might take different value 35, 36, 37, 12, in String Theory and in Loop Quantum Gravity. The status of the energy-momentum dispersion relation within these theories is not completely settled (it is much debated particularly in the Loop-Quantum-Gravity literature), but on the basis of our improved Bekenstein argument we can conclude that both String Theory and Loop Quantum Gravity cannot allow terms of the type $\alpha_{1} E^{3}$ in the energy-momentum dispersion relation. And, if their log-area corrections to the entropy-area relation are to be trusted, we expect that both String Theory and Loop Quantum Gravity should either predict a $\alpha_{2} E^{4}$ correction to the dispersion relation or (see later) they should host a corresponding modification of the positionmomentum uncertainty principle.

We can also use (12) to obtain, using the first law of black hole thermodynamics $d S=\frac{d M}{T}$, a Planck-scale-corrected relation between black-hole temperature and mass:

$$
T_{B H}^{M D R} \simeq \frac{E_{p}^{2}}{8 \pi M}\left(1-\alpha_{1} \frac{E_{p}}{2 \sqrt{2} M}-\left(\frac{15}{32} \alpha_{1}^{2}-\frac{3}{8} \alpha_{2}\right) \frac{E_{p}^{2}}{M^{2}}\right),
$$

where we also used the familiar relation between black hole area and mass $A=16 \pi M^{2}$.

\subsection{Some all-order results for MDR modifications of black-hole entropy}

In the previous subsection we were establishing a possible relation between MDR and log corrections to the entropy-area relation. Since the log-area term is a leading-order term it was appropriate to work within a power-series expansion of the MDR. Moreover, the mentioned results from quantumgravity research (primarily from Loop Quantum Gravity and approaches based on noncommutative geometry) that provide motivation for a Planck-scale modification of the dispersion relation in most cases are obtained within analyses that only have access to the first terms in a power-series expansion of the dispersion relation. Still for some aspects of our analysis it will be useful to contemplate some illustrative examples of all-order dispersion relations, especially when we try to figure out what could be some examples of implications of a MDR for the behaviour of black holes of Planck-length size.

The careful reader can easily verify that once a given energy-momentum dispersion relation $E=$ $f_{\text {disp }}(p)$ is adopted the steps of the calculation reported in the preceding subsection can be followed rather straightforwardly, obtaining

$$
\frac{d S}{d A} \simeq \frac{\min (\Delta S)}{\min (\Delta A)} \simeq \frac{1}{2 L_{p}^{2}} \sqrt{\frac{\pi}{A}} \frac{1}{f_{d i s p}\left(\sqrt{\frac{4 \pi}{A}}\right)}
$$

and

$$
T_{B H} \simeq \frac{1}{4 \pi} f_{\text {disp }}\left(\frac{E_{p}^{2}}{2 M}\right) .
$$

As illustrative examples of "all-order MDRs" we consider the following three cases:

$$
\begin{aligned}
\cosh \left(E / E_{p}\right)-\cosh \left(m / E_{p}\right)-\frac{p^{2}}{2 E_{p}^{2}} e^{E / E_{p}} & =0, \\
\frac{E^{2}}{\left(1-E / E_{p}\right)^{2}}-\frac{p^{2}}{\left(1-E / E_{p}\right)^{2}}-m^{2} & =0, \\
\cosh \left(\sqrt{2} E / E_{p}\right)-\cosh \left(\sqrt{2} m / E_{p}\right)-\frac{p^{2}}{E_{p}^{2}} \cosh \left(\sqrt{2} E / E_{p}\right) & =0,
\end{aligned}
$$

(17) has already been considered in the previous literature [7, 19, 20, 26], particularly as a possible description of particle propagation in $\kappa$-Minkowski noncommutative spacetime. It provides an example in which the coefficient of the linear-in- $L_{p}$ term is nonvanishing: $\alpha_{1}=-1 / 2$. And it is noteworthy 
that according to (17) there is a maximum momentum for fundamental particles: from (17) it follows that for $E \rightarrow \infty$ one has $p \rightarrow E_{p}$.

The case (19) has not been previously considered in the literature. It provides for our purposes a valuable illustrative example since, as in the case of (17), it would lead to a maximum momentum $\left(p \rightarrow E_{p}\right.$ for $\left.E \rightarrow \infty\right)$ but, contrary to the case of (17), it corresponds to $\alpha_{1}=0$ (whereas $\left.\alpha_{2}=-5 / 18\right)$. This is therefore an example with the maximum-momentum feature and such that one would expect the leading corrections to the entropy-area relation to be logarithmic.

The case of (18) has already been considered in the literature for other reasons [21], and it provides us an opportunity to illustrate some consequences of a scenario in which both $\alpha_{1}$ and $\alpha_{2}$ vanish, but still there are some Planck-scale modifications of the energy-momentum dispersion relation. And it is noteworthy that (18) can be implemented in such a way that 21] the Planck scale provides the maximum value of both momentum and energy.

For the cases with dispersion relations (17) or (19), since $E \rightarrow \infty$ for $p \rightarrow E_{p}$, the formulas derived above would lead to the conclusion that the black hole temperature diverges at some finite (nonzero!) value of the black-hole mass $M_{\min }=E_{p} / 2$. We would then assume that this $M_{\min }$ is the minimum allowed mass for a black hole, and that the standard description of the evaporation process should not be applicable beyond this small value of mass.

In cases in which one introduces both a maximum momentum and a maximum energy while keeping the form of the dispersion relation largely unaffected ${ }^{3}$, as done in some applications of (18), one would expect (since the energy has a maximum Planckian value, $E_{M a x}=E_{P}$ ) that the temperature should be bounded to be lower than the Planck scale, $T_{M a x} \sim E_{p}$, and that the minimum allowed value of black-hole mass should be also Plankian, since it should be the value of mass such that temperature reaches is maximum allowed value.

\section{GUP (with and without MDR) and black hole entropy}

In the previous section we focused on scenarios in which the energy-momentum dispersion relation is modified but the position-momentum uncertainty principle preserves the Heisenberg form. But clearly the key ingredient of our analysis is the presence of a correction term $\Delta$ in the particle-localizationlimit relation $E \geq 1 / \delta x+\Delta$. As stressed in Section 2, both a MDR and a GUP can introduce such a correction term in the particle-localization limit, and therefore, as we want to discuss explicitly in this Section, also in presence of a GUP one should expect corrections to the entropy-area black-hole formula and to the formula that relates the mass and the temperature of a black hole.

We start the section by considering scenarios in which the position-momentum uncertainty principle is Planck-scale modified, while the energy-momentum dispersion relation preserves its specialrelativistic form. Then in Subsection 4.2 we comment on the more general case, in which one might be dealing with both a MDR and a GUP.

\subsection{GUP and black hole entropy}

Let us start by noting here again for convenience the particle-localization limit that one obtains assuming a GUP of the form (2) and a standard (special-relativistic) energy-momentum dispersion relation:

$$
E \geq \frac{1}{\delta x}+\alpha \frac{L_{p}^{2}}{(\delta x)^{3}}+O\left(\frac{L_{p}^{3}}{(\delta x)^{4}}\right) .
$$

Following the same strategy of analysis adopted in the previous section, one finds that the Bekenstein argument, when taking into account this localization limit (20), leads to the conclusion that the

\footnotetext{
${ }^{3}$ Whenever the mass $m$ can be ignored (i.e. for massless particles and high-energy particles with finite mass) the dispersion relation (18) is indistinguishable from the standard special-relativistic one.
} 
maximum increase of black-hole area upon absorption of a particle of energy $E$ is given by

$$
\Delta A \geq 4(\ln 2)\left[L_{p}^{2}+\frac{\alpha L_{p}^{4}}{(\delta x)^{2}}\right] \simeq 4(\ln 2)\left[L_{p}^{2}+\frac{\alpha L_{p}^{4}}{\left(R_{S}\right)^{2}}\right] \simeq 4(\ln 2)\left[L_{p}^{2}+\frac{\alpha 4 \pi L_{p}^{4}}{A}\right]
$$

From this it follows that the entropy-area relation should take the form

$$
S \simeq \frac{A}{4 L_{p}^{2}}-\alpha \pi \ln \frac{A}{L_{p}^{2}}
$$

and the formula relating the temperature and the mass of the black hole should take the form

$$
T_{B H}^{G U P} \simeq \frac{E_{p}^{2}}{8 \pi M}\left(1+\alpha \frac{E_{p}^{2}}{8 M^{2}}\right)
$$

\subsection{Combining MDR and GUP in the analysis of black-hole entropy}

We have argued that both a MDR and a GUP are possible features of a quantum-gravity theory that would affect black-hole termodynamics. Actually, as the careful reader must have noticed, the line of analysis we are advocating is composed of two steps. First we notice that the "particle-localization limit" in its standard form, $E \geq 1 / \delta x$, is derived on the basis of two key assumptions, the validity of the Heisenberg position-momentum uncertainty principle and the validity of the special-relativistic energy-momentum dispersion relation, and that by modifying the uncertainty principle and/or the dispersion relation one gets a modified particle-localization limit of the type $E \geq 1 / \delta x+\Delta_{\delta x, L_{p}}$. Then we observe that a key assumption of the Bekenstein argument for the derivation of black-hole entropy is the validity of the standard particle-localization limit $E \geq 1 / \delta x$. With a MDR and/or a GUP one gets a modified particle-localization limit, which in turn leads to a modification of the black-hole area-entropy relationship.

It is worth mentioning that the modifications induced by a MDR and a GUP may (at least in part) cancel out at the level of the area-entropy equation. In order to stress the importance of this possibility let us consider the information presently available on the Loop Quantum Gravity approach: (i) several Loop-Quantum-Gravity studies have argued in favour of a MDR with nonvanishing $\alpha_{1}$ (leading Planckscale correction to the dispersion relation that goes linearly with $L_{p}$ ), (ii) there is no mention of a GUP in the Loop-Quantum-Gravity literature, (iii) several Loop-Quantum-Gravity studies have argued in favour of an entropy-area relationship in which the leading correction, beyond the linear term, is of logarea type. According to the perspective on the derivation of black-hole entropy that we are advocating one would find these three ingredients to be logically incompatible: if the MDR has nonvanishing $\alpha_{1}$ and the position-momentum uncertainty principle is not Planck-scale modified then in the entropyarea relationship the leading correction, beyond the linear term, should have $\sqrt{\text { area }}$ dependence. Does this mean that Loop Quantum Gravity is a logically inconsistent framework? Of course, it does not. It simply means that some of the relevant preliminary results must be further investigated. It may well be that, as the loop-quantum-gravity approach is understood more deeply, it turns out that the $\alpha_{1}$ coefficient in the MDR vanishes. Or else we might discover that in Loop Quantum Gravity the $\alpha_{1}$ coefficient in the MDR takes a nonzero value, but there is a corresponding linear-in- $L_{p}$ term in the GUP with just the right coefficient to give an overall vanishing coefficient to the $\sqrt{\text { area }}$ term in the entropy-area relation.

Our perspective on the derivation of black-hole entropy provides a logical link between different aspects of a quantum-gravity theory and may be used most fruitfully when, as in the case of Loop Quantum Gravity, the formalism is very rich and some of the results obtained within that formalism are of preliminary nature. Even before being able to derive more robust results we may uncover that the presently-available preliminary results are not providing us with a logically-consistent picture, and this in turn will give us additional motivation for investigating more carefully those preliminary results. 
It is also worth mentioning that on the string-theory side our perspective on the derivation of black-hole entropy provides no evidence of a logical inconsistency among the results so far obtained in that framework. The string-theory literature indicates that the entropy-area relationship should involve a leading correction, beyond the linear term, of log-area type, and provides strong evidence of a GUP of the type (2), while the results so far obtained do not indicate the need to modify the dispersion relation in string theory. These three ingredients provide a logically-consistent scenario within our perspective on the derivation of black-hole entropy. As shown above, with a GUP of the type (2) and with an unmodified (still special-relativistic) dispersion relation one is indeed led to an entropy-area relationship in which the leading correction, beyond the linear term, is of log-area type.

\section{Implications for the Bekenstein entropy bound and Generalized Second Law}

It is natural at this point, after having shown that a MDR and a GUP can affect the black-hole entropy-area and mass-temperature relationships, to wonder whether other aspects of black-hole thermodynamics are also affected, and whether the overall picture preserves the elegance/appeal of the original scheme, based on standard uncertainty principle and dispersion relation. In this section we investigate the validity of the Generalized Second Law (GSL) of thermodynamics and the implications for the Bekenstein entropy bound. In order to work within a definite scenario we assume here a MDR (while we implicitly assume that the uncertainty principle takes its standard form).

The GSL 38] asserts that the second law of thermodynamics is still valid in presence of collapsed matter. Given the entropy of the black hole, as described by the area-entropy relation, the GSL requires that the total entropy of a system composed of a black hole and ordinary matter never decreases. This means that the following inequality holds for all physical processes

$$
S_{B H}+S_{m a t} \geq 0 .
$$

It was observed 39] that in principle (using the so-called "Geroch process") one could violate the GSL if objects of fixed size $R$ and energy $E$ could have arbitrarily large entropy $S$. This led Bekenstein to propose a "entropy bound"

$$
S_{\text {mat }} \leq 2 \pi E R
$$

for an arbitrary system of energy $E$ and effective radius $R$. The fact that the GSL implies the Bekenstein bound and vice versa has long been debated and is still actively debated. However the Bekenstein bound turns out to hold for a variety of systems in flat Minkowski space and can be derived as weak-gravity limit of the popular "Generalized Covariant Entropy Bound" [0].

A remarkable feature of the Bekenstein bound is that, in spite of being motivated by considerations rooted in the gravitational realm, it does not involve the Planck scale (or equivalently Newton's constant). The absence of the Planck scale is less puzzling in light of the observation that the bound can be derived even without advocating gravity in any way: it is sufficient [15] to analyze some implications of the particle-localization limit $E \geq \frac{1}{\delta x}$. This alternative derivation requires considering a matter system with energy $E$, in which self-gravitation effects can be neglected, that occupies a region in flat spacetime with radius $R$ smaller than the gravitational radius $R_{G} \equiv 2 L_{p}^{2} E$. The standard particle-localization limit, when generalized to this type of systems, sets a minimum value for the energy of a quantum in a region of spatial radius $R$

$$
\epsilon(R) \geq \frac{1}{R}
$$

The maximum number of quanta that we can have in the region is then given by

$$
N_{\max } \simeq \frac{E}{\epsilon(R)}=E R
$$


If we consider the simple case of a system for which the maximal number of microstates for $N$ particles is given by $\Omega(N)=2^{N}$ then the entropy of the system $S=\log \Omega(N)$ is bounded by the inequality

$$
S_{m a t} \leq \log 2 E R
$$

which is indeed consistent with the Bekenstein bound (up to another "calibration factor" $\eta=\frac{2 \pi}{\log 2}$ ).

We briefly reviewed this derivation of the Bekenstein bound especially in order to stress the role played by the particle-localization limit $E \geq \frac{1}{\delta x}$. It is then obvious that the modifications of the particle-localization limit induced by a MDR (and/or a GUP) would affect the Bekenstein bound. As shown earlier, within our parametrization of the $\mathrm{MDR}^{4}$, one obtains a particle localization limit of the form

$$
\epsilon(R) \geq \frac{1}{R}\left(1-\alpha_{1} \frac{L_{p}}{R}-\left(\frac{3}{2} \alpha_{2}-\frac{11}{8} \alpha_{1}^{2}\right) \frac{L_{p}^{2}}{R^{2}}+O\left(\frac{L_{p}^{3}}{R^{3}}\right)\right)
$$

which gives

$$
S_{m a t} \leq 2 \pi E R\left(1+\alpha_{1} \frac{L_{p}}{R}+\left(\frac{3}{2} \alpha_{2}-\frac{11}{8} \alpha_{1}^{2}\right) \frac{L_{p}^{2}}{R^{2}}+O\left(\frac{L_{p}^{3}}{R^{3}}\right)\right) .
$$

This MDR-modified Bekenstein bound fits very naturally with our corresponding formula, (12), for the entropy-area relation; in fact, the two results combine to provide us with a picture which is still consistent with the GSL. According to (29) when a matter system of energy $E$ falls into the black hole, this corresponds to a negative change of entropy which has absolute value not greater than

$$
\max \left(\left|\Delta S_{\text {mat }}\right|\right) \simeq 2 \pi E R\left(1+\alpha_{1} \frac{L_{p}}{R}+\left(\frac{3}{2} \alpha_{2}-\frac{11}{8} \alpha_{1}^{2}\right) \frac{L_{p}^{2}}{R^{2}}+O\left(\frac{L_{p}^{3}}{R^{3}}\right)\right)
$$

and correspondingly, according to (12), the black hole entropy increases at least by

$$
\min \left(\Delta S_{B H}\right) \simeq 2 \pi E R\left(1+\alpha_{1} \frac{L_{p}}{R}+\left(\frac{3}{2} \alpha_{2}-\frac{11}{8} \alpha_{1}^{2}\right) \frac{L_{p}^{2}}{R^{2}}+O\left(\frac{L_{p}^{3}}{R^{3}}\right)\right)
$$

Thus the MDR-induced corrections to $S_{B H}$ and $S_{\text {mat }}$ cancel exactly at the level of the inequality relevant for the GSL. The GSL stills holds, even in presence of a modified particle-localization limit.

\section{Corrections to black-body radiation spectrum}

In preparation for some observations on black-hole evaporation, to which we devote Section 7, we now want to investigate the implications of a MDR and/or a GUP for the black-body radiation spectrum.

\subsection{MDR and black-body spectrum in leading order}

Let us start by considering photons in a cubical box with edges of length $L$ (and volume $V=L^{3}$ ). The wavelengths of the photons are subject to the boundary condition $\frac{1}{\lambda}=\frac{n}{2 L}$, where $n$ is a positive integer. This condition implies, assuming that the de Broglie relation is left unchanged, that the photons have (space-)momenta that take values $p=\frac{n}{2 L}$. Thus momentum space is divided into cells of volume $V_{p}=\left(\frac{1}{2 L}\right)^{3}=\frac{1}{8 V}$. From this it follows that the number of modes with momentum in the interval $[p, p+d p]$ is given by

$$
g(p) d p=8 \pi V p^{2} d p .
$$

\footnotetext{
${ }^{4}$ For an analogous modification of the Bekenstein bound coming from the GUP see Ref. 15.
} 
Assuming a MDR of the type parametrized in (1) one then finds that ( $m=0$ for photons)

$$
p \simeq E\left(1+\frac{\alpha_{1}}{2} L_{p} E+\left(\frac{\alpha_{2}}{2}-\frac{\alpha_{1}^{2}}{8}\right) L_{p}^{2} E^{2}\right)
$$

and

$$
d p \simeq\left(1+\alpha_{1} L_{p} E+\left(\frac{3}{2} \alpha_{2}-\frac{3}{8} \alpha_{1}^{2}\right) L_{p}^{2} E^{2}\right) d E
$$

Using this in (32) one obtains

$$
g(E) d E=8 \pi V\left(1+2 \alpha_{1} L_{p} E+5\left(\frac{1}{2} \alpha_{2}+\frac{1}{8} \alpha_{1}^{2}\right) L_{p}^{2} E^{2}\right) E^{2} d E
$$

which in terms of the frequency $\nu$ takes the form

$$
g(\nu) d \nu=8 \pi V\left(1+2 \alpha_{1} L_{p} \nu+5\left(\frac{1}{2} \alpha_{2}+\frac{1}{8} \alpha_{1}^{2}\right) L_{p}^{2} \nu^{2}\right) \nu^{2} d \nu
$$

In order to obtain the MDR-modified energy density of a black body at temperature $T$ we must now use (36) and rely on the statistical arguments which show that in a system of bosons at temperature $T$ the average energy per oscillator is given by

$$
\bar{E}=\frac{\nu}{e^{\frac{\nu}{T}}-1} .
$$

Thus the energy density at a given temperature $T$, for the frequency interval $[\nu, \nu+d \nu]$, is

$$
u_{\nu}(T) d \nu=8 \pi\left(1+2 \alpha_{1} L_{p} \nu+5\left(\frac{1}{2} \alpha_{2}+\frac{1}{8} \alpha_{1}^{2}\right) L_{p}^{2} E^{2}\right) \frac{\nu^{3} d \nu}{e^{\frac{\nu}{T}}-1} .
$$

and integrating this formula we get the MDR-modified energy density of a black body at temperature $T$

$$
u(T)=\frac{8 \pi^{5}}{15} T^{4}+384 \pi \zeta(5) \alpha_{1} L_{p} T^{5}+5\left(\frac{1}{2} \alpha_{2}+\frac{1}{8} \alpha_{1}^{2}\right) \frac{160 \pi^{7}}{63} L_{p}^{2} T^{6}
$$

The MDR introduces corrections of the type $T^{4+n} / E_{P}^{n}$ to the Stefan-Boltzmann law. Moreover, the maximum value of the integrand in (38), as a function of $\nu$, is clearly also shifted: the MDR also introduces a modification of Wien's law. Of course, using the low-energy expansion (11) of the dispersion relation we only get a reliable picture at temperatures safely below the Planck scale, but the presence of correction terms of the type $T^{4+n} / E_{P}^{n}$ clearly suggests that the MDR-modified description leads to departures from the Stefan-Boltzmann law that can become very significant as the temperature approaches the Planck scale. We intend to show this explicitly by considering an example of all-order MDR formula.

\subsection{Some all-order results for MDR modifications of black-body spectrum}

Let us therefore derive once again the modified Stefan-Boltzmann law, now assuming, as illustrative example of an all-order MDR formula, the validity of the dispersion relation (19). Clearly the number of modes in momentum space is still given by

$$
g(p) d p=8 \pi V p^{2} d p
$$

but now

$$
p^{2}=E_{p}^{2}\left(1-\frac{1}{\cosh \left(\sqrt{2} E / E_{p}\right)}\right)
$$


and this implies that the number of modes for given energy is given by

$$
g(E) d E=16 \pi V E_{p}^{2} \sinh ^{2}\left(\frac{E / E_{p}}{\sqrt{2}}\right) \cosh \left(\frac{E / E_{p}}{\sqrt{2}}\right) \frac{1}{\cosh ^{5 / 2}\left(\sqrt{2} E / E_{p}\right)} d E
$$

i.e. the number of modes for given frequency is

$$
g(\nu) d \nu=16 \pi V E_{p}^{2} \sinh ^{2}\left(\frac{\nu / E_{p}}{\sqrt{2}}\right) \cosh \left(\frac{\nu / E_{p}}{\sqrt{2}}\right) \frac{1}{\cosh ^{5 / 2}\left(\sqrt{2} \nu / E_{p}\right)} d \nu .
$$

Then the modified Stefan-Boltzmann law is given, in integral form, by

$$
u(T)=\frac{1}{V} \int_{0}^{\infty} \frac{g(\nu)}{e^{\frac{\nu}{T}}-1} \nu d \nu
$$

where $g(\nu)$ is the one of (43).

It is useful to consider some limiting forms of the integration in (44). Clearly, since (19) is consistent with (11) for $\alpha_{1}=0$ and $\alpha_{2}=-5 / 18$, in the limit $T / E_{p} \ll 1$ the integration (44) gives a result that reproduces (39) for $\alpha_{1}=0$ and $\alpha_{2}=-5 / 18$. But, now that we are dealing with an all-order formula, besides considering the case $T / E_{p} \ll 1$ we can also investigate the opposite limit $T / E_{p} \gg 1$, finding

$$
u(T)=16 \pi E_{p}^{4}\left\{\frac{T}{E_{p}} C_{1}-\frac{1}{2} C_{2}-\frac{E_{p}}{T} C_{3}+O\left(E_{p}^{2} / T^{2}\right)\right\}
$$

where

$$
\begin{aligned}
& C_{1}=\int_{0}^{\infty} \sinh ^{2}(x / \sqrt{2}) \frac{\cosh (x / \sqrt{2})}{\cosh ^{5 / 2}(\sqrt{2} x)} d x=\frac{1}{6}, \\
& C_{2}=\int_{0}^{\infty} x \sinh ^{2}(x / \sqrt{2}) \frac{\cosh (x / \sqrt{2})}{\cosh ^{5 / 2}(\sqrt{2} x)} d x \simeq 0.22, \\
& C_{3}=\int_{0}^{\infty} x^{2} \sinh ^{2}(x / \sqrt{2}) \frac{\cosh ^{2}(\sqrt{2})}{\cosh ^{5 / 2}(\sqrt{2} x)} d x \simeq 0.41,
\end{aligned}
$$

This means that the MDR (19) leads to a modification of the Stefan-Boltzmann law which at the Planck scale is very significant: for $T \gg E_{p}$ one finds that $u$ depends linearly on $T$, rather than with the fourth power.

It is of particular interest to establish what is the relationship between the "characteristic frequency" (and characteristic wavelength) of the black-body spectrum and temperature. In the standard description of a black body the characteristic frequency grows linearly with the temperature. In order to verify whether this is still the case in our MDR-modified scenario we can take the derivative of $u_{\nu}(T)$ with respect to $\nu$, so that we can identify the value of frequency for which the energy density (and the radiated flux) reaches a maximum. This leads to the following equation that must be satisfied by the characteristic frequency $\bar{\nu}$ :

$$
\left(e^{\frac{\bar{\nu}}{T}}-1\right)\left(g(\bar{\nu})+g^{\prime}(\bar{\nu}) \bar{\nu}\right)-\frac{e^{\frac{\bar{\nu}}{T}}}{T} g(\bar{\nu}) \bar{\nu}=0 .
$$

For $T \ll E_{p}$ of course this reproduces the type of small modification of Wien's law, which we already noticed in the previous section. The fact that we are now considering a scenario with a given all-order MDR formula allows us to examine the dependence of the characteristic frequency on temperature even when the temperature reaches and eventually exceeds the Planck scale. And we find that the for 
$T / E_{p} \gg 1$ the characteristic frequency becomes essentially independent of temperature. No matter how high the temperature goes the characteristic frequency never exceeds the following finite value:

$$
\bar{\nu} \simeq E_{p} \frac{\cosh ^{-1}[(1+\sqrt{41}) / 4]}{\sqrt{2}} \simeq 0.87 E_{p}
$$

So basically at low temperatures any increase of temperature causes a corresponding increase in characteristic frequency of the black-body spectrum, but gradually a saturation mechanism takes over and even in the infinite-temperature limit the characteristic frequency is still finite, and given by the Planck scale (up to a coefficient of order 1). This occurs with the dispersion relation (19), i.e. in a scenario with a minimum value of wavelength but no maximum value of frequency. An analogous result for the case of the dispersion relation (18), which leads to both a minimum value of wavelength and a maximum value of frequency, would have not been surprising: if the framework introduces from the beginning a maximum Planckian value of frequency, then of course also the characteristic frequency of black-body radiation would be "subPlanckian". But in analyzing the case of (19) we found that the presence of a minimum wavelength at the fundamental level is sufficient for the emergence of a maximum Planckian value of the characteristic frequency of black-body radiation, as shown explicitly by Eq. (50).

\subsection{Black-body spectrum with GUP}

In the previous two subsections the key point was that a MDR leads to a modified formula for the density of modes in a given (infinitesimal) frequency interval, $g(\nu) d \nu$. If instead we now assume that the dispersion relation takes its standard special-relativistic form, but there is a GUP, it is not $a$ priori obvious that the black-body spectrum is affected. One does indeed obtain a modified blackbody spectrum if it is assumed that the GUP should also be reflected in a corresponding modification of the de Broglie relation,

$$
\lambda \simeq \frac{1}{p}\left(1+\alpha L_{p}^{2} p^{2}\right)
$$

and

$$
E \simeq \nu\left(1+\alpha L_{p}^{2} \nu^{2}\right)
$$

For oscillators in a box the number of modes in an infinitesimal frequency interval would still be described by the standard formula

$$
g(\nu) d \nu=8 \pi V \nu^{2} d \nu
$$

but, as a result of (52), the average energy per oscillator would be given by

$$
\bar{E}=\frac{\nu}{e^{\frac{\nu}{T}}-1}\left(1+\alpha L_{p}^{2} \nu^{2}\left(1-\frac{\frac{\nu}{T}}{1-e^{-\frac{\nu}{T}}}\right)\right) .
$$

Combining (52) and (54) one finds

$$
u_{\nu}(T) d \nu=8 \pi\left(1+\alpha L_{p}^{2} \nu^{2}\left(1-\frac{\frac{\nu}{T}}{1-e^{-\frac{\nu}{T}}}\right)\right) \frac{\nu^{3} d \nu}{e^{\frac{\nu}{T}}-1} .
$$

and the modified Stefan-Boltzmann law takes the form

$$
u(T)=\frac{8 \pi^{5}}{15} T^{4}+\frac{8 \pi^{6}}{9} \alpha L_{p}^{2} T^{6} .
$$

The $L_{p}^{2} T^{6}$ correction term is just one of the $L_{p}^{n} T^{4+n}$ correction terms on which we already commented in the context of the MDR modifications of black-body radiation. 


\section{Black hole evaporation}

In this section we use some of the results obtained in the previous sections in a description of the blackhole evaporation process. The key ingredients are the relation between the black-hole temperature and mass and the relation between the black-hole temperature and the energy density emitted by the black hole. We neglect possible non-thermal corrections due to back-reaction effects (see, e.g., the recent studies in Ref. [41, 42] and references therein), and we therefore treat the radiation emitted by the black-hole as black-body radiation.

\subsection{MDR and Black hole evaporation}

At temperature $T$ the intensity $I$ of the radiation emitted by a black hole of area $A$ is given by

$$
I(T)=A u(T) .
$$

Using energy conservation one can write

$$
\frac{d M}{d t}=-A u
$$

and assuming a MDR of the type $E=f_{\text {disp }}(p)$, in light of our result (16), one finds

$$
\frac{d M}{d t}=-16 \pi \frac{M^{2}}{E_{p}^{4}} u\left(\frac{1}{4 \pi} f_{d i s p}\left(\frac{E_{p}^{2}}{2 M}\right)\right)
$$

When $M \gg E_{p}$ (so that a power-series expansion of $f_{d i s p}\left(E_{p}^{2} / 2 M\right)$ is meaningful) this takes the form

$$
\frac{d M}{d t}==-k_{0} \frac{E_{p}^{8}}{M^{2}}-k_{1} \alpha_{1} \frac{E_{p}^{9}}{M^{3}}-\left(k_{21} \alpha_{1}^{2}+k_{22} \alpha_{2}\right) \frac{E_{p}^{10}}{M^{4}}+O\left(E_{p}^{5} / M^{5}\right)
$$

where $k_{0}=\frac{\pi^{2}}{480}, k_{1}=k_{0} \frac{90 \zeta(5)-\pi^{5}}{\pi^{5}}, k_{21}=k_{0} \frac{502 \pi^{5}-75600 \zeta(5)}{672 \pi^{5}}$ and $k_{22}=-k_{0} \frac{211}{672 \pi^{5}}$

This power-series analysis allows to conclude that a MDR can affect the speed of evaporation of a black hole. For example, in the case of the dispersion relation (17) the evaporation process is retarded with respect to the standard case, whereas in the case of (19) the evaporation process is accelerated.

With a given all-order MDR formula one can obtain of course even more detailed information than available using the power-series expansion. In particular, let us look at the case of the dispersion relation (19) and analyze the stage of the evaporation process when the mass of the black hole is of the order of the Planck scale. For $M \sim E_{P}$ we can approximate the MDR (19) as follows

$$
E \simeq \frac{E_{p}}{\sqrt{2}} \ln \left(\frac{2}{1-\left(p / E_{p}\right)^{2}}\right)
$$

and then one finds

$$
\frac{d M}{d t} \simeq-(16 \pi)^{2} M^{2}\left\{\frac{C_{1}}{4 \pi \sqrt{2}} \ln \left(\frac{2}{1-\left(\frac{E_{p}}{2 M}\right)^{2}}\right)-\frac{1}{2} C_{2}\right\} .
$$

This shows that, in the case of the MDR (19), the energy flux emitted by the black hole would formally diverge as the black-hole mass approaches $E_{p} / 2$. This is mainly a consequence of the fact that the black-hole temperature diverges when $M \rightarrow E_{p} / 2$. In the standard description of black-hole evaporation these divergences occur as $M \rightarrow 0$. 


\subsection{GUP and Black hole evaporation}

The observations reported in the previous subsection for the case of a MDR (with unmodified energymomentum uncertainty relation) can be easily adapted to the complementary situation with a GUP and a standard (unmodified) dispersion relation. One must however assume, as already stressed in Subsection 6.3, that the GUP is reflected in a corresponding modification of the de Broglie relation $\left(\lambda \simeq\left(1+\alpha L_{p}^{2} p^{2}\right) / p\right)$. In this hypothesis one easily finds that the black hole should lose its mass at a rate given by

$$
\frac{d M}{d t}=-A u\left(\frac{E_{p}^{2}}{2 M}\right)=-16 \pi \frac{8 \pi^{5}}{15}\left(T\left(\frac{E_{p}^{2}}{2 M}\right)\right)^{4}+\frac{8 \pi^{6}}{9} \alpha L_{p}^{2}\left(T\left(\frac{E_{p}^{2}}{2 M}\right)\right)^{6} .
$$

Expanding for $M / E_{p} \gg 1$ we obtain

$$
\frac{d M}{d t} \simeq-16 \pi \frac{E_{p}^{4}}{M^{2}}\left(\tilde{k}_{0}+\alpha \tilde{k}_{1} \frac{E_{p}^{2}}{M^{2}}\right)
$$

with $\tilde{k}_{0}=\frac{\pi}{7680}$ and $\tilde{k}_{1}=\frac{1}{294912}+\frac{\pi}{15360}$.

Clearly the modifications to the black hole evaporation formula obtained in the GUP scenario are qualitatively the same as in the MDR scenario with $\alpha_{1}=0$.

\section{A possible dependence on the speed law for photons}

Throughout our analysis we have implicitly assumed that the law $v_{\gamma}=1$ describing the speed of photons is not affected by the MDR and/or the GUP. The possibility of modifications of the speed law for photons has been however considered rather extensively, particularly in the MDR literature. While several authors have argued that the law $v_{\gamma}=1$ should not be modified even in presence of an MDR (see, e.g., Refs. 43, 44, 45] and references therein), one also finds support in the literature for the proposal (see, e.g., Ref. [26] and references therein) of the law $v_{\gamma}=[d E / d p]_{m=0}=\left[d f_{d i s p}(p) / d p\right]_{m=0}$ and the proposal (see, e.g., Ref. 21] and references therein) of the law $v_{\gamma}=p / E$.

For our analysis a key point is that if, instead of $v_{\gamma}=1$, one took $v_{\gamma}=[d E / d p]_{m=0}$ or $v_{\gamma}=p / E$ then the speed of photons would acquire an energy dependence which should be taken into account in some aspects of our derivations. We postpone to future studies this more general analysis, but in order to explore the type of modifications which could be induced by such an energy dependence of the speed of photons we do intend to consider here the description of black-body radiation with the dispersion relation (19), assuming that the speed of photons is governed by either $v_{\gamma}=[d E / d p]_{m=0}$ or $v_{\gamma}=p / E$.

We focus on the emitted "flux density"

$$
I_{\nu}=A u_{\nu} v_{\gamma}(\nu)
$$

where $A$ is the area of the radiating surface and $u_{\nu}$ is the energy density at a given frequency.

Taking $v_{\gamma}=p / E$, from (19) it follows that

$$
v_{\gamma}(\nu)=\frac{p}{E}=\frac{E_{p}}{E} \sqrt{1-\frac{1}{\cosh \left(\frac{\sqrt{2} E}{E_{p}}\right)}} .
$$

From this it would then follow that the energy flux density is given by

$$
I_{\nu}(T)=4 \pi A \sqrt{2} E_{p}^{3} \frac{1}{e^{\nu / T}-1} \frac{\sinh \left(\sqrt{2} E / E_{p}\right)}{\cosh ^{3}\left(\sqrt{2} E / E_{p}\right)}\left[\cosh \left(\sqrt{2} E / E_{p}\right)-1\right] .
$$


This suggests that, although there are some small quantitative differences, the qualitative features of black-body radiation with the dispersion relation (19) are largely independent of the choice between $v_{\gamma}=1$ and $v_{\gamma}=p / E$. In particular, from (67) with one finds that the typical frequency of the photons contributing to the energy flux saturates at

$$
\bar{\nu} \simeq 0.76 E_{p},
$$

which is not much different from the typical frequency found for the case $v_{\gamma}=1$. The analysis of the total emitted energy $\left(\int_{0}^{\infty} I_{\nu}(T) d \nu\right)$ also leads to rather small differences between the choices $v_{\gamma}=1$ and $v_{\gamma}=p / E$. In particular from (67) one finds

$$
I / A=\frac{8}{15} \pi^{5} T^{4}\left\{1+C_{1}\left(\frac{T}{E_{p}}\right)^{2}+C_{2}\left(\frac{T}{E_{p}}\right)^{4}+O\left(\frac{T}{E_{p}}\right)^{6}\right\},
$$

in the limit $T / E_{p} \ll 1$, and

$$
I / A=E_{p}^{4}\left\{\widetilde{C}_{1} \frac{T}{E_{p}}+\widetilde{C}_{2}+\widetilde{C}_{3} \frac{E_{p}}{T}+O\left(\frac{E_{p}}{T}\right)^{2}\right\},
$$

in the limit $T / E_{p} \gg 1$, where $C_{1}=-\frac{100 \pi^{2}}{21}, C_{2}=\frac{164 \pi^{4}}{5}$ and $\widetilde{C}_{1}=5.57, \widetilde{C}_{1}=-\pi$ and $\widetilde{C}_{3}=0.79$.

If instead one adopts the law $v_{\gamma}=[d E / d p]_{m=0}$, still assuming (19), one obtains

$$
v_{\gamma}(\nu)=\frac{d E}{d p}=\frac{\cosh \frac{\frac{3}{2}}{\frac{\sqrt{2} E}{E_{p}}}}{\cosh \frac{E}{\sqrt{2} E_{p}}}
$$

and then the flux density takes the form

$$
I_{\nu}=16 \pi A E_{p}^{2} \nu \frac{\sinh ^{2} \frac{\nu}{\sqrt{2} E_{p}}}{\left(e^{\frac{\nu}{T}}-1\right) \cosh \frac{\sqrt{2} \nu}{E_{p}}} .
$$

From this one easily verifies that the effects induced by the Planck-scale deformation in the case $v_{\gamma}=[d E / d p]_{m=0}$ are essentially of the same type encountered in the cases $v_{\gamma}=1$ and $v_{\gamma}=p / E$, but the quantitative differences between the case $v_{\gamma}=[d E / d p]_{m=0}$ and the other two cases are more significant then the ones between the cases $v_{\gamma}=1$ and $v_{\gamma}=p / E$. As mentioned, in absence of the Planck-scale effects the typical frequency of the photons contributing to the energy flux grows linearly with the temperature, while in the cases in which the Planck-scale effects of (19) are introduced with $v_{\gamma}=1$ or $v_{\gamma}=p / E$ the typical frequency saturates at a Planckian value. If the same Planck-scale effects are introduced with $v_{\gamma}=[d E / d p]_{m=0}$, as implicitly codified in (72), one finds that the growth of the typical frequency with temperature also slows down significantly at high temperatures but it does not completely saturate: at high temperatures the typical frequency grows logarithmically with the temperature.

In summary the choice of the speed law does not appear to affect the core features of the analysis, but it appears that it could in some cases introduce some significant quantitative differences.

\section{Comparison with previous analyses}

To our knowledge, the one we reported here, in spite of its preliminary nature, is at this point the most composite effort of exploration of the implications of a MDR and/or a GUP in black-hole thermodynamics. But parts of the overall picture we attempted to provide had been investigated previously, and it seems appropriate to comment briefly on this previous related studies. 
Closest in spirit to our perspective are the studies of the implications of the GUP for black-hole thermodynamics reported in Refs. [14] and [15]. Whereas for us (2) is to be handled prudently, as it could possibly be only an approximate form of a more complicated all-order-in- $L_{p}$ formula, in Refs. [14, 15] the formula (2) is taken as the exact form of the GUP, thereby leading to a corresponding form of the entropy-area relation. Perhaps more importantly Refs. 14, 15] assume that the GUP would not affect the black-body spectrum and in particular a standard expression for Stefan's law is used even in Planckian regimes. There was no investigation of MDRs in Refs. [14, 15].

An attempt to describe Hawking radiation in presence of a MDR was reported in Ref. 13. There the problem is approached from the field-theoretic perspective, considering possible modification of the field equations coming from the MDR. No explicit formula for the corrections to the Hawking spectrum and to the entropy-area relation was obtained in Ref. 13.

Ref. [16] investigates how a general form of the GUP could modify the volume element of phase space, and therefore the black-body-radiation formula, using the Hamiltonian formulation in terms of Poisson brackets.

In Ref. [17] an analysis of black-body radiation is carried out in presence of a MDR of the type emerging from a proposed "semiclassical limit" of Loop Quantum Gravity, which is analogous to the "leading order" MDR (11) we studied in some parts of this paper. The results reported there are consistent with the power-series formulas for Stefan's and Wien's law which we derived. The features we exposed in considering some illustrative examples of all-order MDRs, were not discussed in Ref. [17. Also the entropy-area relation and the aspects of black-hole evaporation which we considered here were not part of the analysis reported in Ref. [17, and Ref. [17 did not consider the possibility of a GUP.

Ref. [18] is closest in spirit to the part of our analysis where we focused on the black-body radiation spectrum, as affected by a MDR. Although the formal setup differs in several points, the results are roughly consistent with ours, including the possibility of "saturation" of the characteristic frequency at $T \gg E_{p}$. There was however no investigation of the entropy-area relation and the Generalized Second law in Ref. [18, and Ref. [18] also did not consider the possibility of a GUP.

\section{Outlook}

The technical difficulties that are encountered in most approaches to the quantum-gravity problem usually only allow one to grasp a few disconnected aspects of the physical picture that the theories could provide. And in some approaches even the few "physical" results that are obtained, are only derived within approximation schemes whose reliability is not fully established. We have argued that in this situation it might be particularly valuable to establish a few logical links connecting some apparently unrelated aspects of the physical picture. And we showed that such a link can be found between some aspects of quantum-gravity research which have attracted strong interest in recent times, a link providing a connection between results on modified energy-momentum dispersion relations and/or modified position-momentum uncertainty principles and results on the thermodynamics of black holes. We have provided a description of log corrections to the entropy-area law for black holes that is based on the availability of a MDR and/or a GUP.

In exploring other aspects of black-hole thermodynamics as affected by MDRs and GUPs we stumbled upon a few noteworthy points. We found that the Generalized Second Law of thermodynamics might be robust enough to survive the introduction of these Planck-scale effects. We found that a MDR introducing a minimum value for wavelengths (even when no maximum value for frequencies is introduced) could lead to a description of black-body radiation in which the characteristic frequency of the radiation never exceeds a finite Planckian value (described in Eq. (50)). This in turn also affects black-hole evaporation in such a way that the temperature diverges already when the mass of the black hole decreases to a Planck-scale value (instead of diverging only in the zero-mass limit as usually assumed).

A key test for our line of analysis will come from future improved analyses within the loop-quantumgravity approach. According to the perspective we adopted some preliminary results on the emergence of modifications of the dispersion relation that depend linearly on the Planck length (at low energies) would be incompatible with the loop-quantum-gravity results on log corrections to the entropy-area 
relation for black holes. We predict that improved analyses of the loop-quantum-gravity approach should lead to the emergence of a picture that is instead compatible with the conceptual link we are proposing.

As stressed in Section 8 one aspect of our analysis in which we took a rather conservative attitude (in comparison with the possibilities considered in the literature) is the one concerning the description of the speed of photons, which we assumed to be still frequency independent. We do not expect major obstacles for a generalization of our analysis with the inclusion of the possibility of a frequencydependent speed of photons, and the preliminary investigation reported in Section 8 suggests that some of the core features that emerged from our analysis are only moderately affected by the choice of law for the speed of photons.

\section{Acknowledgments}

The work of M. A. was supported by a Fellowship from The Graduate School of The University of North Carolina. M. A. also thanks the Department of Physics of the University of Rome for hospitality. Y. L. is partly supported by NSFC (No.10205002,10405027) and SRF for ROCS,SEM.

\section{References}

[1] G. Amelino-Camelia, J. Ellis, N.E. Mavromatos, D.V. Nanopoulos and S. Sarkar, astro-ph/9712103, Nature 393 (1998) 763.

[2] G. Amelino-Camelia, gr-qc/0309054

[3] L. Smolin, hep-th/0303185.

[4] D. Mattingly, gr-qc/0502097

[5] R. Gambini and J. Pullin, Phys. Rev. D59 (1999) 124021; J. Alfaro, H.A. Morales-Tecotl and L.F. Urrutia, Phys. Rev. Lett. 84 (2000) 2318.

[6] G. Amelino-Camelia, L. Smolin and A. Starodubtsev, hep-th/0306134.

[7] J. Lukierski, H. Ruegg and W.J. Zakrzewski, Ann. Phys. 243 (1995) 90.

[8] M. Maggiore, Phys. Lett. B 304, 65 (1993) arXiv:hep-th/9301067.

[9] L. J. Garay, Int. J. Mod. Phys. A 10, 145 (1995) arXiv:gr-qc/9403008.

[10] G. Veneziano, Europhys. Lett. 2 (1986) 199; D.J. Gross and P.F. Mende, Nucl. Phys. B303 (1988) 407; D. Amati, M. Ciafaloni and G. Veneziano, Phys. Lett. B216 (1989) 41.

[11] S. Doplicher, K. Fredenhagen and J.E. Roberts, Phys. Lett. B331 (1994) 39.

[12] G. Amelino-Camelia, M. Arzano and A. Procaccini, Int. J. Mod. Phys. D13 (2004) 2337; gr-qc/0405084 Phys. Rev. D70 (2004) 107501.

[13] A. Blaut, J. Kowalski-Glikman and D. Nowak-Szczepaniak, Phys. Lett. B 521, 364 (2001) arXiv:gr-qc/0108069.

[14] R. J. Adler, P. Chen and D. I. Santiago, Gen. Rel. Grav. 33, 2101 (2001) arXiv:gr-qc/0106080.

[15] P. S. Custodio and J.E. Horvath, Class. Quant. Grav. 20 (2003) L197.

[16] L. N. Chang, D. Minic, N. Okamura and T. Takeuchi, Phys. Rev. D 65, 125028 (2002) arXiv:hep-th/0201017.

[17] H. Yepez, J. M. Romero and A. Zamora, arXiv:hep-th/0407072

[18] S. Alexander and J. Magueijo, hep-th/0104093. 
[19] G. Amelino-Camelia, gr-qc/0012051, Int. J. Mod. Phys. D11 (2002) 35.

[20] J. Kowalski-Glikman and S. Nowak, hep-th/0204245, Int. J. Mod. Phys. D12 (2003) 299.

[21] J. Magueijo and L. Smolin, gr-qc/0207085 Phys. Rev. D67 (2003) 044017.

[22] T. Kifune, Astrophys. J. Lett. 518 (1999) L21.

[23] R. Aloisio, P. Blasi, P.L. Ghia and A.F. Grillo, Phys. Rev. D62 (2000) 053010.

[24] G. Amelino-Camelia and T. Piran, astro-ph/0008107, Phys. Rev. D64 (2001) 036005.

[25] T. Jacobson, S. Liberati and D. Mattingly, hep-ph/0112207, Phys. Rev. D66 (2002) 081302.

[26] G. Amelino-Camelia, F. D'Andrea and G. Mandanici: hep-th/0211022 JCAP 0309 (2003) 006.

[27] J. D. Bekenstein, Phys. Rev. D7 (1973) 2333.

[28] D. Christodoulou, Phys. Rev. Lett. 25 (1970) 1596; D. Christodoulou and R. Ruffini, Phys. Rev. D4 (1971) 3552.

[29] S. Hod, Phys. Rev. Lett. 81 (1998) 4293.

[30] P. Chen and R.J. Adler, Nucl. Phys. Proc. Suppl. 124 (2003) 103.

[31] T. Damour, hep-th/0401160 M. Cavaglia, S. Das and R. Maartens, Class. Quant. Grav. 20 (2003) L205.

[32] E. M. Lifshitz, L. P. Pitaevskii and V. B. Berestetskii, "Landau-Lifshitz Course of Theoretical Physics, Volume 4: Quantum Electrodynamics" (Reed Educational and Professional Publishing, 1982).

[33] M.Yu. Kuchiev and V.V. Flambaum, gr-qc/0312065

[34] R.J. Adler and T.K. Das, Phys. Rev. D14 (1976) 2472; R.S. Hanni and R. Ruffini, "Lines of Force of a point Charge Near a Schwarzschild Black Hole", in Black Holes, eds. C. DeWitt and B.S. DeWitt (Gordon Breach 1973).

[35] A. Strominger and C. Vafa, Phys. Lett. B379 (1996) 99; S. N. Solodukhin, Phys. Rev. D57 (1998) 2410.

[36] C. Rovelli, Phys. Rev. Lett. 77 (1996) 3288; A. Ashtekar, Phys. Rev. Lett. 80 (1998) 904; R.K. Kaul and P. Majumdar, Phys. Rev. Lett. 84 (2000) 5255.

[37] G. Gour and A.J.M. Medved, Class. Quant. Grav. 20 (2003) 3307; S. Das, hep-th/0403202

[38] J. D. Bekenstein, Phys. Rev. D 9, 3292 (1974).

[39] J. D. Bekenstein, Phys. Rev. D 23, 287 (1981).

[40] R. Bousso, JHEP 0405, 050 (2004) arXiv:hep-th/0402058.

[41] M. Arzano, hep-th/0504188.

[42] A.J.M. Medved and E.C. Vagenas, gr-qc/0505015.

[43] P. Kosinski and P. Maslanka, hep-th/0211057, Phys. Rev. D68 (2003) 067702.

[44] S. Mignemi, hep-th/0302065 Phys. Lett. A316 (2003) 173.

[45] M. Daszkiewicz, K. Imilkowska and J. Kowalski-Glikman, "Velocity of particles in doubly special relativity," arXiv:hep-th/0304027 